\title{
Spin-wave series for quantum one-dimensional ferrimagnets
}

\author{
N. B. Ivanov \\ Institute for Solid State Physics, Bulgarian Academy of Sciences, \\ Tzarigradsko chaussee-72, 1784 Sofia, Bulgaria
}

(April 10, 2018)

\begin{abstract}
Second-order spin-wave expansions are used to compute the ground-state energy and sublattice magnetizations of the quantum one-dimensional Heisenberg ferrimagnet with nearest-neighbor antiferromagnetic interactions and two types of alternating sublattice spins $S_{1}>S_{2}$. It is found that in the extreme quantum cases $\left(S_{1}, S_{2}\right)=(1,1 / 2),(3 / 2,1)$, and $(3 / 2,1 / 2)$, the estimates for the ground-state energy and sublattice magnetizations differ less than $0.03 \%$ for the energy and $0.2 \%$ for the sublattice magnetizations from the recently published density matrix renormalization group numerical calculations. The reported results strongly suggest that the quantum Heisenberg ferrimagnetic chains give another example of a low-dimensional quantum spin system where the spin-wave approach demonstrates a surprising efficiency.

PACS: 75.50.Ee, 75.10.Jm, 75.30.Kz, 75.10.-b
\end{abstract}

Typeset using REVTEX 
Recently, two scientific groups have published theoretical results concerning the model of the one-dimensional Heisenberg ferrimagnet (1DQHF) containing two alternating site spins $\left(S_{1}>S_{2}\right)$ per unitary cell and nearest-neighbor antiferromagnetic bonds [1] 4 ]. The presented linear spin-wave analysis demonstrates a substantial reduction of the classical sublattice spins imposed by the quantum fluctuations. In the extreme quantum cases with $\left(S_{1}, S_{2}\right)=(1,1 / 2),(3 / 2,1)$, and $(3 / 2,1 / 2)$, the quantum reduction of $S_{2}$ was shown to be about $61 \%, 46 \%$, and $37 \%$, respectively. The density matrix renormalization group results [4] point towards a smaller reduction. The cited values are respectively reduced to $42 \%, 36 \%$, and 28\%. For comparison, in the square-lattice Heisenberg antiferromagnet the discussed reduction is about $39 \%$. It is clear that the linear theory overestimates the role of the zeropoint spin fluctuations. In this respect, an open question is if the qualitative picture based on the LSWT (and concerning, in particular, the structure of the elementary excitations) can reflect the real situation at all. The purpose of the present paper is to throw some light on the above problem through an explicit study of the large $S$ series for the ground state energy and sublattice magnetizations up to second order in $1 / S$.

The Hamiltonian of the 1DQHF with two spins $S_{1}>S_{2}$ per unitary cell and nearestneighbor antiferromagnetic couplings reads

$$
H=\sum_{n, \delta} \mathbf{S}_{\mathbf{1}_{n}} \mathbf{S}_{\mathbf{2} n+\delta},
$$

where the intergers $n$ number the cells and the vector $\delta= \pm 1 / 2$ connects the two nearestneighbor spins. The size of the elementary cell and the exchgange interaction are unities. In what follows we frequently use the notations $S_{1} / S_{2} \equiv w, S_{2} \equiv S$.

We will use the Dyson-Maleev representation for the site spin operators. After some standard procedures [5], including the Fourier and Bogoliubov transformations and the normal ordering of the boson operators, the spin Hamiltonian can be recasted to the following form

$$
H=H_{0}+V, \quad V=c_{1}+V_{2}+V_{D M},
$$




$$
\begin{aligned}
H_{0} & =\left(-2 w S^{2}+2 S c_{0}\right) N+2 S \sum_{k}\left[\omega_{k}^{(\alpha)} \alpha_{k}^{\dagger} \alpha_{k}+\omega_{k}^{(\beta)} \beta_{k}^{\dagger} \beta_{k}\right] \\
V_{2} & =\sum_{k}\left[V_{k}^{(1)} \alpha_{k}^{\dagger} \alpha_{k}+V_{k}^{(2)} \beta_{k}^{\dagger} \beta_{k}+V_{k}^{(3)} \alpha_{k}^{\dagger} \beta_{k}^{\dagger}+V_{k}^{(4)} \alpha_{k} \beta_{k}\right]
\end{aligned}
$$

where $c_{0}=-\left[1-(1 / N) \sum_{k} \epsilon_{k}\right](w+1) / 2, c_{1}=-2\left(g_{1}^{2}+g_{2}^{2}\right)-2 g_{1} g_{2}(w+1) w^{-1 / 2}, g_{1}=$ $-(1 / 2 N) \sum_{k} \gamma_{k} \eta_{k} / \epsilon_{k}, g_{2}=-(1 / 2)+(1 / 2 N) \sum_{k} 1 / \epsilon_{k}, \epsilon_{k}=\left(1-\eta_{k}^{2}\right)^{1 / 2}, \eta_{k}=2 \gamma_{k} w^{1 / 2} /(w+1)$, $\gamma_{k}=\cos (k / 2)$, and $N$ is the number of cells.

$H_{0}$ is the quadratic LSWT Hamiltonian. The boson operators $\alpha_{k}$ and $\beta_{k}$ describe two types of elementary excitations with energies

$$
E_{k}^{(\alpha, \beta)}=2 S \omega_{k}^{(\alpha, \beta)}=2 S\left[\frac{w+1}{2} \epsilon_{k} \mp \frac{(w-1)}{2}\right] .
$$

The $\alpha$ excitations are gapless $\left(\omega_{k}^{(\alpha)} \sim k^{2}\right.$ for small $\left.k\right)$ and describe magnons in the sector with a total spin $\left(S_{1}-S_{2}\right) N-1$, whereas the $\beta$ excitations are gapful $\left(\omega_{k}^{(\beta)}=w-1+O\left(k^{2}\right)\right)$ and belong to the sector $\left(S_{1}-S_{2}\right) N+1$.

The interaction $V$ contains three different terms: The constant $c_{1}$ gives the first-order correction to the ground state energy. The quadratic interaction $V_{2}$ introduces four vertex functions defined as follows

$$
\begin{gathered}
V_{k}^{(1)}=-\frac{g_{1}}{\epsilon_{k}}\left(\frac{w+1}{\sqrt{w}}-2 \gamma_{k} \eta_{k}+\frac{w-1}{\sqrt{w}} \epsilon_{k}\right)-\frac{g_{2}}{\epsilon_{k}}\left(2-\frac{w+1}{\sqrt{w}} \gamma_{k} \eta_{k}\right) \\
V_{k}^{(3)}=-\frac{g_{1}}{\epsilon_{k}}\left(2 \gamma_{k}-\frac{w+1}{\sqrt{w}} \eta_{k}\right)-\frac{g_{2}}{\epsilon_{k}}\left(\frac{w+1}{\sqrt{w}} \gamma_{k}+\frac{w-1}{\sqrt{w}} \gamma_{k} \epsilon_{k}-2 \eta_{k}\right) \\
V_{k}^{(4)}=V_{k}^{(3)}+2 g_{2} \frac{w-1}{\sqrt{w}} \gamma_{k}, V_{k}^{(2)}=V_{k}^{(1)}-2 g_{1} \frac{w-1}{\sqrt{w}} .
\end{gathered}
$$

For the model under consideration the off-diagonal terms of $V_{2}$ do not vanish due to the inequality $S_{1}>S_{2}$. Note also that $V_{2}$ is a non-Hermitian operator for the same reason.

$V_{D M}$ is the Dyson-Maleev normal ordered quartic interaction, containing nine vertex functions $V^{(i)}=V_{12 ; 34}^{(i)}, i=1, \ldots, 9$. We have adopted the symmetric form used in Refs. [6,7] and the convention $\left(k_{1}, k_{2}, k_{3}, k_{4}\right) \equiv(1,2,3,4)$. 
Now, let us represent the series for the ground state energy and the magnetization of the first sublattice in the following form

$$
\begin{gathered}
\frac{E_{0}}{N}=-2 w S^{2}+2 S c_{0}+c_{1}+\frac{c_{2}}{2 S}+\ldots \\
m_{1}=w S+b_{0}+\frac{b_{1}}{2 S}+\frac{b_{2}}{(2 S)^{2}}+\ldots
\end{gathered}
$$

The coefficient $b_{0}=-g_{2}$ gives the spin reduction in the LSWT. The first-order correction for $m_{1}$ is related to the off-diagonal terms in $V_{2}$, and a simple calculation gives the following result

$$
b_{1}=-\frac{1}{2(w+1)} \frac{1}{N} \sum_{k}\left(V_{k}^{(3)}+V_{k}^{(4)}\right) \frac{\eta_{k}}{\epsilon_{k}^{2}}
$$

The coefficients $c_{2}$ and $b_{2}$ will be calculated using the Rayleigh-Schrodinger perturbation theory. A straightforward calculation gives the following two contributions to $c_{2}=c_{21}+c_{22}$ resulting from $V_{2}$ and $V_{D M}$, respectively

$$
c_{21}=-\frac{1}{w+1} \frac{1}{N} \sum_{k} \frac{V_{k}^{(3)} V_{k}^{(4)}}{\epsilon_{k}}, \quad c_{22}=-\frac{2}{w+1} \frac{1}{N^{3}} \sum_{1-4} \delta_{12}^{34} \frac{V_{12 ; 34}^{(7)} V_{34 ; 12}^{(8)}}{\epsilon_{1}+\epsilon_{2}+\epsilon_{3}+\epsilon_{4}} .
$$

. Here $\delta_{12}^{34}$ is the Kronicker function. The symmetric vertex functions $V_{12 ; 34}^{(7)}$ and $V_{12 ; 34}^{(8)} \operatorname{read}$

$$
\begin{aligned}
V_{12 ; 34}^{(7)} & =\mathcal{U}_{1234}\left\{x_{1}\left[x_{4}\left(\gamma_{1-3}-w^{-1 / 2} x_{3} \gamma_{1}\right)-\left(w^{1 / 2} \gamma_{1-3-4}-x_{3} \gamma_{1-4}\right)\right]\right. \\
& \left.+x_{2}\left[x_{4}\left(\gamma_{2-3}-w^{-1 / 2} x_{3} \gamma_{2}\right)-\left(w^{1 / 2} \gamma_{2-3-4}-x_{3} \gamma_{2-4}\right)\right]\right\}, \\
V_{12 ; 34}^{(8)} & =\mathcal{U}_{1234}\left\{x_{2}\left[\left(x_{3} \gamma_{1-3}-w^{-1 / 2} \gamma_{1}\right)-x_{4}\left(w^{1 / 2} x_{3} \gamma_{1-3-4}-\gamma_{1-4}\right)\right]\right. \\
& \left.+x_{1}\left[\left(x_{3} \gamma_{2-3}-w^{-1 / 2} \gamma_{2}\right)-x_{4}\left(w^{1 / 2} x_{3} \gamma_{2-3-4}-\gamma_{2-4}\right)\right]\right\},
\end{aligned}
$$

where $\mathcal{U}_{1234}=u_{1} u_{2} u_{3} u_{4}, u_{k}=\left(1+\epsilon_{k}\right) / 2 \epsilon_{k}$, and $x_{k}=\eta_{k} /\left(1+\epsilon_{k}\right)$.

To calculate $b_{2}$ we introduce in $H$ a staggered magnetic field for $S_{1}$ spins through the operator $-h_{1} \sum_{n} S_{1 n}^{z}$. Then the required second-order correction for $m_{1}$ can be deduced from $m_{1}^{(2)}=-\left.(1 / N)\left[\partial E_{0}^{(2)}\left(h_{1}\right) / \partial h_{1}\right]\right|_{h_{1}=0}$, where $E_{0}^{(2)}\left(h_{1}\right)$ is the second-order correction to $E_{0}$ in a finite $h_{1}$. As a matter of fact, $h_{1}$ reduces the structure factor to $\eta_{k} \longmapsto \eta_{k}=$ 
$2 \gamma_{k} w^{1 / 2} /\left(1+w+h_{1} / 2 S\right)$, and this is all one needs to find all necessary derivatives. In some cases, it is better to use an infinitesimal perturbing field $h_{1}$ and the related perturbed ground state $\left|0_{h_{1}}\right\rangle=|0\rangle+\left[h_{1} / 2 S(w+1)\right] \sum_{k}\left(\eta_{k} / \epsilon_{k}^{2}\right) \alpha_{k}^{\dagger} \beta_{k}^{\dagger}|0\rangle+O\left(h_{1}^{2}\right)$.

Using the latter approach, one can easily find [7] two of the contributions to $b_{2}=b_{21}+$ $b_{22}+b_{23}+b_{24}$ related to the interaction $V_{D M}$

$$
\begin{gathered}
b_{21}=-\frac{2}{(w+1)^{2}} \frac{1}{N^{3}} \sum_{1-4} \delta_{12}^{34} \frac{V_{12 ; 34}^{(7)} V_{34 ; 12}^{(8)}}{\left(\epsilon_{1}+\epsilon_{2}+\epsilon_{3}+\epsilon_{4}\right)^{2}}\left(\frac{1}{\epsilon_{1}}+\frac{1}{\epsilon_{2}}+\frac{1}{\epsilon_{3}}+\frac{1}{\epsilon_{4}}\right), \\
b_{22}=\frac{2 w^{1 / 2}}{(w+1)^{3}} \frac{1}{N^{3}} \sum_{1-4} \delta_{12}^{34} \frac{W_{12 ; 34}}{\epsilon_{1}+\epsilon_{2}+\epsilon_{3}+\epsilon_{4}}, \\
W_{12 ; 34}=V_{12 ; 34}^{(7)}\left[\frac{\gamma_{2}}{\epsilon_{2}^{2}} V_{34 ; 12}^{(5)}+\frac{\gamma_{3}}{\epsilon_{3}^{2}} V_{34 ; 12}^{(2)}\right]+\left[\frac{\gamma_{1}}{\epsilon_{1}^{2}} V_{12 ; 34}^{(6)}+\frac{\gamma_{4}}{\epsilon_{4}^{2}} V_{12 ; 34}^{(3)}\right] V_{34 ; 12}^{(8)} .
\end{gathered}
$$

The vertex functions appearing in the last equation have the same structure as $V_{12 ; 34}^{(7)}$ and $V_{12 ; 34}^{(8)}$ and we will not present here their explicit expressions.

For the other two contributions to $b_{2}$, which are related to the interaction $V_{2}$, we use the first of the mentioned methods. The result reads

$$
b_{23}=-\frac{1}{(w+1)^{2}} \frac{1}{N} \sum_{k} \frac{V_{k}^{(3)} V_{k}^{(4)}}{\epsilon_{k}^{3}}, \quad b_{24}=\frac{1}{(w+1)^{2}} \frac{1}{N} \sum_{k} \frac{1}{\epsilon_{k}}\left[U_{k}^{(3)} V_{k}^{(4)}+U_{k}^{(4)} V_{k}^{(3)}\right],
$$

where $U_{k}^{(3)}=-2 g_{1} \gamma_{k} / \epsilon_{k}-2 g_{2}\left(1-\gamma_{k}^{2}\right) \eta_{k} / \epsilon_{k}^{3}-g_{3}\left[(w+1) w^{-1 / 2} \gamma_{k}+(w-1) w^{-1 / 2} \gamma_{k} \epsilon_{k}-2 \eta_{k}\right] / \epsilon_{k}$, $U_{k}^{(4)}=U_{k}^{(3)}+2 g_{3}(w-1) w^{-1 / 2} \gamma_{k}, g_{3}=-(1 / 2 N) \sum_{k} \eta_{k}{ }^{2} / \epsilon_{k}{ }^{3}$. Notice that the equation $m_{1}+$ $m_{2}=S_{1}-S_{2}$, which is connected to the conservation law for the total magnetization, is fulfilled order by order in the spin-wave series, so that it is enough to know only the corrections for one of the sublattice magnetizations.

The results for the series for a number of combinations $\left(S_{1}, S_{2}\right)$ are presented in the tables. Surprisingly, even in the extreme quantum cases $(3 / 2,1)$ and $(1,1 / 2)$, the deviations from the density matrix renormalization group results are less than $0.033 \%$ for the energy and $0.2 \%$ for the sublattice magnetizations. One can also notice that the insrease of the ratio $w=S_{1} / S_{2}$ for fixed $S_{2} \equiv S=1 / 2$ leads to a rapid improvement of the series. This 
tendency is expected because for large $w$ the quasiclassical spins on the first sublattice act as an effective field on the $S_{2}=1 / 2$ spins.

It is instructive to compare the series for 1DQHF with those for the square-lattice Heisenberg antiferromagnet (2DQHA) [8]. Let us take the series for $w=2$ and for the second sublattice magnetization $m_{2}$.

$$
\begin{aligned}
1 D Q H F: & \frac{E_{0}}{N}=-4 S^{2}-0.436456 \times(2 S)-0.024384+0.006518 \times \frac{1}{2 S}+\ldots \\
2 D Q H A: & \frac{E_{0}}{N}=-4 S^{2}-0.315895 \times(2 S)-0.024948+0.000866 \times \frac{1}{2 S}+\ldots \\
1 D Q H F: & -m_{2}=S-0.3048865+0.1212303 \times \frac{1}{2 S}-0.0224602 \times \frac{1}{(2 S)^{2}}+\ldots \\
2 D Q H A: & m=S-0.1966019+0 \times \frac{1}{2 S}+0.00348 \times \frac{1}{(2 S)^{2}}+\ldots
\end{aligned}
$$

The $E_{0}$ spin-wave series for the compared models have similar structures.. The $1 / S$ correction in 1DQHF is somewhat larger, but note that the LSWT reduction is also larger for the $1 \mathrm{D}$ model. The values of the coefficients $c_{1}$ are very close to each other. As to the sublattice magnetizations, the main difference comes from the lack of $b_{1}$ corrections in the 2DQHA which is connected with the symmetry of the square lattice. Note that up to the first order the quantum spin reductions in the models are approximately one and the same. As a whole, the spin-wave series for the 1DQHF demonstrate features which are compatible with those of the 2DQHA spin-wave series. It is well-known that the spin-wave results for the ground state parameters of the $S=1 / 2$ square-lattice Heisenberg antiferromagnet are close to the most precise numerical estimates [9]. The reported results strongly suggest that the quantum Heisenberg ferrimagnetic chains give another example of a low-dimensional quantum spin system where the spin-wave approach demonstrates a surprising efficiency.

The author thanks J. Richter for useful discussions and the staff of the Institut für Theoretische Physik for hospitality. The stay in the Universität Magdeburg was supported by DFG. 


\section{REFERENCES}

[1] S. K. Pati, S. Ramasesha, and D. Sen, Phys. Rev. B 55, 8894 (1997).

[2] A. K. Kolezhuk, H.-J. Mikeska, and S. Yamamoto, Phys. Rev. B 55, R3336 (1997).

[3] S. Brehmer, H.-J. Mikeska, and S. Yamamoto, J. Phys.: Condens. Matter 9, 3921 (1997).

[4] S. K. Pati, S. Ramasesha, and D. Sen, J. Phys.: Condens. Matter 9, 8707 (1997).

[5] A. B. Harris, D. Kumar, B. I. Halperin, and P. C. Hohenberg, Phys. Rev. B 3, 961 (1971).

[6] C. M. Canali and S. M. Girvin, Phys. Rev. B 45, 7127 (1992).

[7] N. B. Ivanov, S. E. Krüger, and J. Richter, Phys. Rev. B 53, 2633 (1996).

[8] C. J. Hamer, Z. Weihong, and P. Arndt, Phys. Rev. B 46, 6276 (1992).

[9] A. W. Sandvik, Phys. Rev. B 56, 11678 (1997). 


\section{TABLES}

TABLE I. The coefficients of the spin-wave series for the ground state energy per cell $\epsilon_{0}=E_{0} / N$ of ferrimagnetic chains with two different spins: $S_{1}=w S_{2}, S_{2} \equiv S, w>1$; $\left.\epsilon_{0}=-2 w S^{2}+c_{0}(w) \times(2 S)+c_{1}(w)+c_{2}(w) \times(2 S)^{-1}+O\left[(2 S)^{-2}\right)\right]$

\begin{tabular}{ccccccc}
\hline \hline$\left(S_{1}, S_{2}\right)$ & $w=S_{1} / S_{2}$ & $c_{0}(w)$ & $c_{1}(w)$ & $c_{2}(w)$ & $\epsilon_{0}(\mathrm{SWT})$ & $\epsilon_{0}(\mathrm{DMRG})$ 狛 \\
\hline$\left(\frac{3}{2}, 1\right)$ & 1.5 & $-0.41403688(9)$ & $-0.03950436(9)$ & $0.00874156(4)$ & -3.86320737 & -3.86192 \\
$\left(1, \frac{1}{2}\right)$ & 2 & $-0.43645559(0)$ & $-0.02438442(5)$ & $0.00651791(7)$ & -1.45432210 & -1.45408 \\
$\left(\frac{3}{2}, \frac{1}{2}\right)$ & 3 & $-0.45803557(4)$ & $-0.01179278(6)$ & $0.00283359(4)$ & -1.96699477 & -1.96724 \\
$\left(2, \frac{1}{2}\right)$ & 4 & $-0.46862597(4)$ & $-0.00691029(8)$ & $0.00139788(3)$ & -2.47413839 & \\
$\left(9, \frac{1}{2}\right)$ & 18 & $-0.49305421(4)$ & $-0.00037529(8)$ & $0.00002099(5)$ & -9.49340852 & \\
\hline \hline
\end{tabular}

TABLE II. The coefficients of the spin-wave series for the sublattice magnetization $m_{1}$ of ferrimagnetic chains with two different spins: $S_{1}=w S_{2}, S_{2} \equiv S, w>1$; $\left.m_{1}=w S+b_{0}(w)+b_{1}(w) \times(2 S)^{-1}+b_{2}(w) \times(2 S)^{-2}+O\left[(2 S)^{-3}\right)\right]$

\begin{tabular}{ccccccc}
\hline \hline$\left(S_{1}, S_{2}\right)$ & $w=S_{1} / S_{2}$ & $b_{0}(w)$ & $b_{1}(w)$ & $b_{2}(w)$ & $m_{1}(\mathrm{SWT})$ & $m_{1}(\mathrm{DMRG})$ \\
\hline$\left(\frac{3}{2}, 1\right)$ & 1.5 & $-0.46005842(4)$ & $0.22694904(9)$ & $-0.02899689(7)$ & 1.14616688 & 1.14427 \\
$\left(1, \frac{1}{2}\right)$ & 2 & $-0.30488650(5)$ & $0.12123033(0)$ & $-0.02246016(3)$ & 0.79388366 & 0.79248 \\
$\left(\frac{3}{2}, \frac{1}{2}\right)$ & 3 & $-0.18644025(0)$ & $0.05316804(7)$ & $-0.01006592(1)$ & 1.35666188 & 1.35742 \\
$\left(2, \frac{1}{2}\right)$ & 4 & $-0.13512460(0)$ & $0.03000271(9)$ & $-0.00504066(8)$ & 1.88983745 & \\
$\left(9, \frac{1}{2}\right)$ & 18 & $-0.02818572(2)$ & $0.00152353(6)$ & $-0.00007830(4)$ & 8.97325951 & \\
\hline \hline
\end{tabular}

